\title{
A Multiperiodic Optimization Formulation for the Operation Planning of Free-Floating Shared Bike in China
}

\author{
Meiyu Li ${ }^{D},{ }^{1,2}$ Xifu Wang, ${ }^{1}$ Xi Zhang, ${ }^{1}$ Lifen Yun $\left(\mathbb{D},{ }^{1}\right.$ and Yuan Yuan ${ }^{1}$ \\ ${ }^{1}$ School of Traffic and Transportation, Beijing Jiaotong University, Beijing, China \\ ${ }^{2}$ School of Business, Hebei GEO University, Shijiazhuang, China \\ Correspondence should be addressed to Lifen Yun; yunlifen@bjtu.edu.cn
}

Received 30 March 2018; Accepted 4 September 2018; Published 25 September 2018

Academic Editor: Alessandro De Luca

Copyright (c) 2018 Meiyu Li et al. This is an open access article distributed under the Creative Commons Attribution License, which permits unrestricted use, distribution, and reproduction in any medium, provided the original work is properly cited.

\begin{abstract}
Internet shared bike has trigged a revolution on the public bicycle utilization in China for various characteristics such as freefloating, intelligent unlocking, mobile payment, intelligent integration, and optional serving. It attracts many users and meanwhile accumulates the development of short-distance alternative trip. This paper has designed an optimization model for the deployment and travel of free-floating bike sharing (FFBS) among small regions. For a given demand under the constraint of overload of flexile stations, the model makes a decision on the minimum number of bike deployments in the system and the planning of bike movement between stations at different time periods. It maximizes the profit of operators during the whole planning horizon and meanwhile satisfices the demand and minimizes the overload situation of stations. The proposed approach is verified with numerical example, aiming to help operators to program and manage systems in a more efficient way.
\end{abstract}

\section{Introduction}

Bicycling, as an advantageous traffic mode, has been brought into the sustainable traffic planning of many countries. It not only features low pollution and low energy but also being efficient in reassuring traffic load and developing people's health. Under this background, policies about accumulating and generalizing bicycle can be seen in a dramatic increase. Therefore, shared bike systems have been introduced and generalized in a growing number of counties.

Since China introduced public bicycles in 2007, both the practical and the theoretical research studies have been at the forefront of the international arena. On one hand, China has accumulated amounts of experiences and data of public bicycle systems and the public bicycle program in Hangzhou has been taken as a success example in a long time. On the other hand, China's public bicycle system faces new challenges such as the development of new manufacture, finance, technology, resource, and new problems caused by the difference of time, region, and users. In recent years, people's consumption model upgrades with the deeper development and revolution in the technology area such as smartphone, $4 \mathrm{G}$ Internet, mobile payment, Internet of things (IoT), and APP. An increasing number of people have accepted Internet shared bike because of its operation characteristics such as free-floating, Internet unlocking, mobile payment, intelligent integration, and optional service which solves the first and last-mile problem. The "Report on the development of China's free-floating shared bicycle Industry (2018)" pointed out that, by the end of 2017, the permeability of FFBS reached a new high of $28.6 \%$ with more than 221 million registered users [1]. It makes an economic and social impact on the whole society with 29.947 billion kilometers of cumulative cycling and with more than $\$ 194$ million incomes. At the same time, FFBS has also put forward a revolution on the operational model of the regional public bike. The report shows that the percentage of FFBS replacing driving car is $55 \%[1]$.

In the operation system of FFBS in China, operators take public place as parking sites without any other set up parking place and locking equipment. Users can acquire bikes at any free-floating site near their starting point and return the bike at any public parking place near their destination when finishing riding. Along with the characteristics of convenience and openness of free-floating operation, the free-floating operation system grows into a huge system in China. Taking 
OfO and Mobile as examples, by July 2017, there, respectively, had been nearly 1 million shared bikes necessary to operate in Beijing. The huge and dynamic features of this system make the managing plan especially important. In the managing plan, one of the basic problems is to decide the deployment of the system and the allocation of bikes at different time slots. If the operation situation of the system is not carefully analyzed and reasonably intervened, the allocation of bikes will be unbalanced due to spatiotemporal changes. The unbalance leads to unsatisfied demand at some sites and overload at the other sites and finally affects the successful operation of FFBS. Hence, to make sure the reliability and proper-allocation of bikes at different stations is of great importance to the feasibility of the system.

This paper designs an optimization model for the operation system based on the characteristics of weak dependency on stations and locking-slots in the operation of FFBS in China. With consideration on the demand and overload of flexible sites, a decision is made on the minimum number of bike deployment in the system and the planning of bike movement between sites at different time periods. The model maximizes the profit of operators during the whole planning horizon and meanwhile satisfices the demands as much as possible and minimizes the overload situation of sites.

The rest of the paper will be organized as follows. Section 2 reviews the relevant literature and practice. Section 3 elaborates the problems which may be solved by the model and proposes a nonlinear integer programming formulation for the multiperiodic operation planning and utilization of FFBS. Section 4 practices the model with a numerical example. Section 5 draws a conclusion and discusses the extending study.

\section{Literature Review}

Although extensive studies have been carried out in the literature about station-based bike sharing (SBBS), most of them focus on the spatiotemporal analysis, operation, and dispatching with limited research in fleet dimensioning. George \& Xia's study (2011) was the first to discuss the fleet dimensioning problem in a shared mobility system [2]. Shu et al. (2013) discussed the allocation of bikes in a bicycle sharing network management and highlighted the importance to deploy proper number of bikes at proper sites [3]. Through a series of hypothesis, they turned the random network flow model into a linear model. Fricker \& Gast (2016) used queuing theory to analyze the effects of capacity of bike station on the system performance [4]. Lin \& Yang (2011) and Lin et al. (2013) studied the design of public bike networks based on the service level $[5,6]$. Raviv \& Kolka (2013) and Raviv et al. (2013) studied the sizing problem of a public bike station $[7,8]$. The authors built a dynamic inventory system model, pursuing to minimize the dissatisfaction of users. Their study focused on the analysis of demand situation at different periods and supposed that the demand of a single station follows the process of a nonhomogeneous Poisson. However, their model ignored the interaction between different stations and simplified the problem as an inventory management problem at one single station. With consideration of the relocation cost, Nair \& Miller-Hooks (2011) identified the allocation scale of a shared car system at different sites and time periods to minimize the total cost of allocation and reallocation [9]. Based on the characteristics of satisfying the demand through probability constrained analogy and static scheduling, the authors modeled the problem as a constrained stochastic optimization. Schuijbroek et al. (2017) defined the fill level with Markov Chain and discussed the vehicle routing problem in the bike shared system [10]. Vogel et al (2014) built up a mixed integer programming model to decide the best fill level of stations with the least relocation cost in multiperiodic shared bike systems [11]. That paper, along with the papers of Vogel (2016) and NeumannSaavedra et al. $(2015,2016)$, focused on the design of the service network and the planning of vehicle routing [12-14]. In the study of Sayarshad et al. (2012), the minimal fleet size in the bike shared system of small communities was shown by a mathematic model [15]. The authors discussed the optimization formulation of multiperiodic bike travel and, however, ignored the capacity limitation of stations.

FFBS greatly reduces the system startup cost since it avoids the construction of stations and self-help service terminal equipment as well as the maintenance cost. Few studies were carried out for FFBS as it is a relatively innovative model. Reiss \& Bogenberger (2015) introduced the moving strategy of FFBS systems in Munich through analyzing the GPS data [16]. Weikl \& Bogenberger (2013) studied the static partial rebalancing of free-floating shared car [17]. Pal \& Zhang (2017) and Liu et al. (2018) discussed the vehicle routing problems in a static partial rebalancing of FFBS systems [18, 19]. Caggiani et al. $(2016,2018)$ proposed applying clustering methodology to predict the spatiotemporal tendency of the utilization mode of FFBS systems so as to improve the relocation of FFBS [20, 21]. Caggiani et al. (2017) proposed using the income of congestion pricing to cover the cost of the FFBS system program [22]. Liu et al. (2018) and Ai et al. (2018) discussed the distribution inference of FFBS systems $[23,24]$.

All the above studies are worthy of using as a reference to the operation of China's FFBS systems. However, a new operation mode faces new problems. Due to the weak dependency on station and locking place of FFBS, market coverage and users' dependence become the most important target for operators in the early stage. Therefore, operators put large amounts of bikes at different sites in the early stage which leads to overload at some sites, causing city management problems and a negative image to the operators. In this paper, a balanced model which realizes the interactional effects among bikes deployment in the multiperiodic operation demands satisfaction and overload at flexible sites.

\section{The Mathematical Formulation}

3.1. Problem Statement. In this part, a multiperiodic optimization formulation of operation planning of FFBS is introduced. Shared bikes in the system are allocated at different sites inside two small regions, and users rent bikes near 


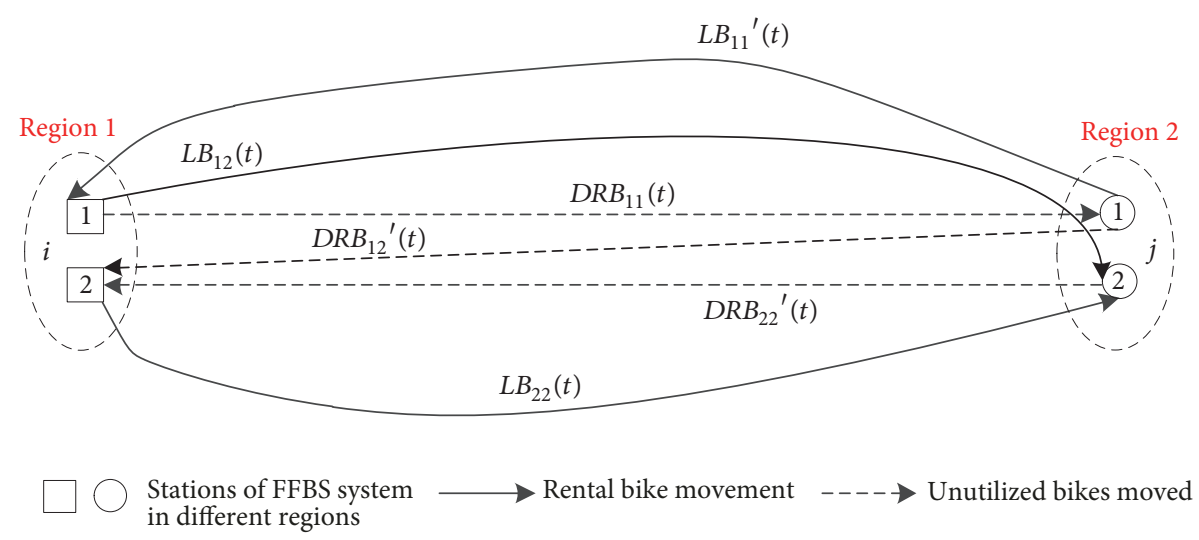

FIGURE 1: Illustration of a FFBS system and movements of rented and empty bikes.

the region of their starting points and return bikes to sites near their destination region. The system charges the users according to the service time. The number of remaining bikes and rented bike at a site in the system can be chased by GPS. Due to the unbalanced demands distribution, human intervention is necessary to move bikes in the overload regions to another region in larger demand. The model proposed in this paper is mainly used to decide a proper bike allocation plan to minimize the situation of dissatisfaction and overload during the whole planning horizon with the smallest system configuration.

\subsection{Premises and Assumption}

(i) In the planning horizon, the total revenue of operators is made up of six parts: bike renting income, empty bike moving cost, holding cost of inventory bikes at sites, purchasing cost, penalty cost when the demand is unsatisfied, and penalty cost when flexible station is overloaded (since flexible site occupies public place with no clear boundary, it brings negative effects to the appearance and environment of a city. Therefore, every site sets a threshold to boundary and once the capacity of a site breaks the threshold, it will be punished).

(ii) Bidirectional trip demand means that one site in a region can be both start point and destination. That is to say that one site in a region can be both trip generating point and trip attracting point. Between two sites, there is no convective transport movement of empty bikes at the same time period, which means that between two sites at different regions moving in and moving out an empty bike cannot be operated at the same time period. In this paper, a region is assumed to be a small region with short distance between two stations. Thus, unutilized bikes will not be moved among different sites at a same small region.

(iii) The whole planning horizon is divided into several time periods. The bidirectional trip demand in each time period is known (it can be acquired from daily average in practice), and the demands will trigger the movement of bikes (bike renting and empty bike moving). The demands that are not satisfied at one time period will be calculated into the next time period, assuming that the users are still in need and waits to be served in the next time period.

(iv) We assume that all rides of bike renting and moving can be accomplished within a single time period. Operators deploy initial number of bikes at the beginning of the planning horizon and then dispatch the empty bike according to the demand situation when the renting is completed in each time period. The empty bikes moving at beginning of current time period (after the bike is rented) will be completed and reach the destination at the end of the current time period, and the bikes will be a part of the initial inventory of the next time period at that station.

(v) The renting and dispatching operation starts from the initial period in the whole planning horizon.

3.3. Mathematical Model. To clearly formulate the optimization problem studied in this paper, we summarize the notations in Table 1.

Figure 1 shows the movement of rented and empty bikes between two stations in an FFBS system. Figure 2 illustrates the dynamic operation process of the multiperiod inventory at each station.

We formulate the objective function as the total benefit of the operator, denoted by the following. The total benefit includes six terms: (i) the revenue from rented bicycles traveling, (ii) transporting cost of empty bikes (including startup and operation cost), (iii) the holding cost at stations, (iv) the purchasing cost, (v) the penalty cost when the demand is not satisfied, and (vi) the penalty cost for overload at stations. With the notations defined in Table 1, the objective function is defined as follows: 


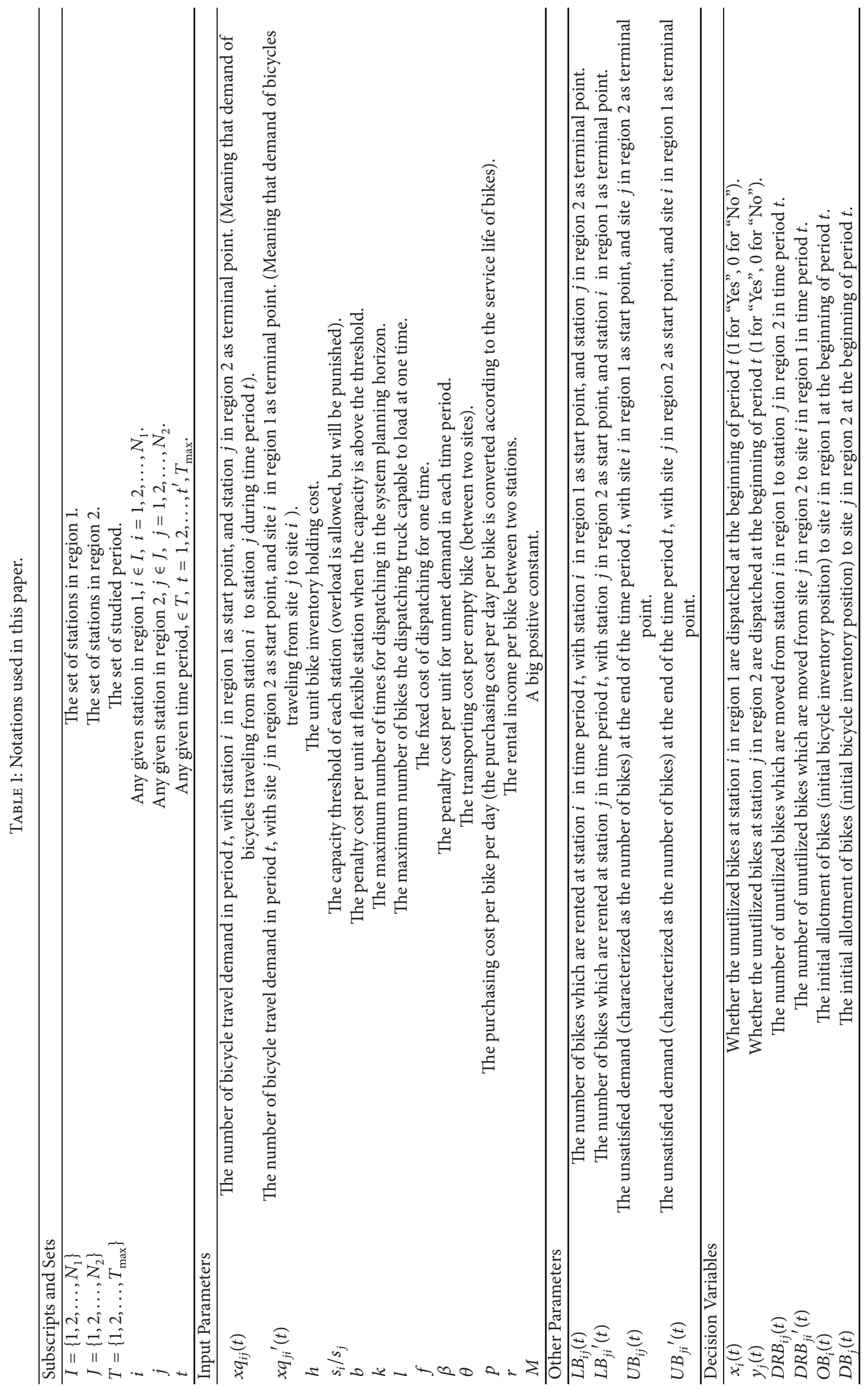




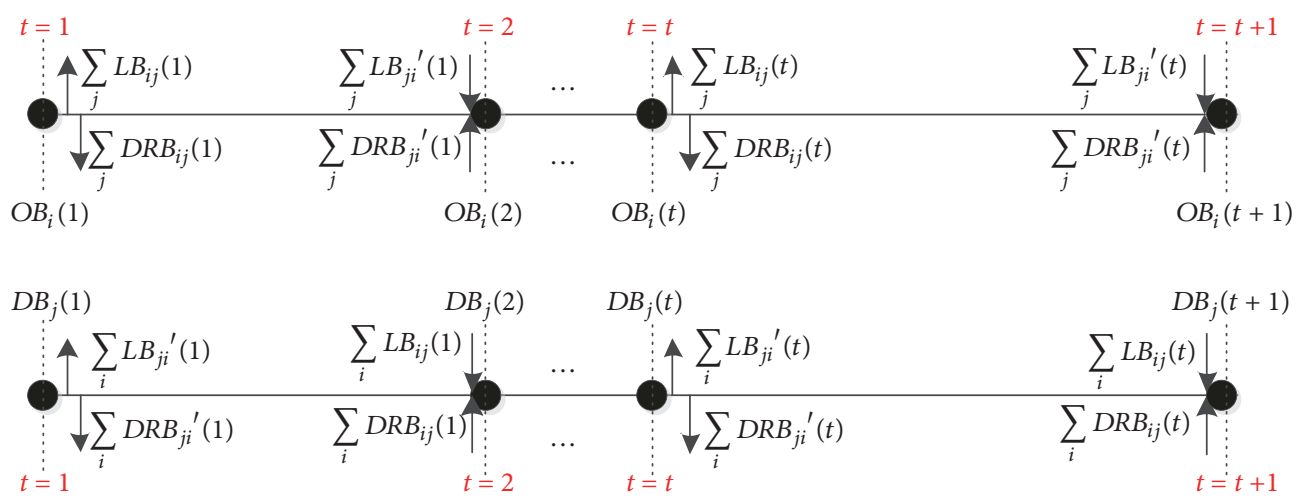

FIGURE 2: Multiperiodic station inventory dynamic models.

$\max \eta$

$$
\begin{aligned}
= & \sum_{i=1}^{N_{1}} \sum_{j=1}^{N_{2}} \sum_{t=1}^{T_{\max }} r \cdot\left[L B_{i j}(t)+L B_{j i}{ }^{\prime}(t)\right]-f\left[\sum_{i=1}^{N_{1}} \sum_{t=1}^{T_{\max }} x_{i}(t)+\sum_{j=1}^{N_{2}} \sum_{t=1}^{T_{\max }} y_{j}(t)\right]-\sum_{i=1}^{N_{1}} \sum_{j=1}^{N_{2}} \sum_{t=1}^{T_{\max }} \theta \cdot\left[x_{i}(t) \cdot D R B_{i j}(t)+y_{j}(t) \cdot D R B_{j i}{ }^{\prime}(t)\right] \\
& -\left\{\sum_{i=1}^{N_{1}} \sum_{t=1}^{T_{\max }} h \cdot\left[O B_{i}(t)-\sum_{j=1}^{N_{2}}\left(L B_{i j}(t)+x_{i}(t) \cdot D R B_{i j}(t)\right)\right]+\sum_{j=1}^{N_{2}} \sum_{t=1}^{T_{\max }} h \cdot\left[D B_{j}(t)-\sum_{i=1}^{N_{1}}\left(L B_{j i}{ }^{\prime}(t)+y_{j}(t) \cdot D R B_{j i}{ }^{\prime}(t)\right)\right]\right\} \\
& -\left[\sum_{i=1}^{N_{1}} p \cdot O B_{i}(1)+\sum_{j=1}^{N_{2}} p \cdot D B_{j}(1)\right]-\sum_{i=1}^{N_{1}} \sum_{j=1}^{N_{2}} \beta \cdot\left[U B_{i j}(t)+U B_{j i}{ }^{\prime}(t)\right] \\
& -\mathrm{b}\left[\sum_{i=1}^{N_{1}} \sum_{t=1}^{T_{\max }} \max \left\{0,\left(O B_{i}(t)-s_{i}\right)\right\}+\sum_{j=1}^{N_{2}} \sum_{t=1}^{T_{\max }} \max \left\{0,\left(D B_{j}(t)-s_{j}\right)\right\}\right]
\end{aligned}
$$

s.t. $\quad O B_{i}(t+1)=O B_{i}(t)-\sum_{j=1}^{N_{2}} L B_{i j}(t)-\sum_{j=1}^{N_{2}} x_{i}(t) \cdot D R B_{i j}(t)+\sum_{j=1}^{N_{2}} L B_{j i}{ }^{\prime}(t)+\sum_{j=1}^{N_{2}} y_{j}(t) \cdot D R B_{j i}{ }^{\prime}(t), \quad \forall i \in I, \forall t \in T \backslash T_{\max }$

$D B_{j}(t+1)=D B_{j}(t)-\sum_{i=1}^{N_{1}} L B_{j i}{ }^{\prime}(t)-y_{j}(t) \sum_{i=1}^{N_{1}} D R B_{j i}{ }^{\prime}(t)+\sum_{i=1}^{N_{1}} L B_{i j}(t)+\sum_{i=1}^{N_{1}} x_{i}(t) \cdot D R B_{i j}(t), \quad \forall j \in J, \quad \forall t \in T \backslash T_{\max }$

$U B_{i j}(t+1)=U B_{i j}(t)+x q_{i j}(t+1)-L B_{i j}(t+1), \quad \forall i \in I, \forall j \in J, \quad \forall t \in T \backslash T_{\max }$

$U B_{j i}{ }^{\prime}(t+1)=U B_{j i}{ }^{\prime}(t)+x q_{j i}{ }^{\prime}(t+1)-L B_{j i}{ }^{\prime}(t+1), \quad \forall i \in I, \quad \forall j \in J, \quad \forall t \in T \backslash T_{\max }$

$U B_{i j}(t)=x q_{i j}(t)-L B_{i j}(t), \quad \forall i \in I, \forall j \in J, \forall t \in T, t=1$

$U B_{j i}{ }^{\prime}(t)=x q_{j i}{ }^{\prime}(t)-L B_{j i}{ }^{\prime}(t), \quad \forall i \in I, \forall j \in J, \forall t \in T, t=1$

$\sum_{j=1}^{N_{2}} L B_{i j}(t) \leq O B_{i}(t) \quad \forall i \in I, \forall t \in T$

$\sum_{i=1}^{N_{1}} L B_{j i}{ }^{\prime}(t) \leq D B_{j}(t) \quad \forall j \in J, \forall t \in T$

$\sum_{j=1}^{N_{2}} D R B_{i j}(t) \leq O B_{i}(t)-\sum_{j=1}^{N_{2}} L B_{i j}(t) \quad \forall i \in I, \quad \forall t \in T$

$\sum_{i=1}^{N_{1}} D R B_{j i}{ }^{\prime}(t) \leq D B_{j}(t)-\sum_{i=1}^{N_{1}} L B_{j i}{ }^{\prime}(t) \quad \forall j \in J, \quad \forall t \in T$ 


$$
\begin{aligned}
& \sum_{j=1}^{N_{2}} D R B_{i j}(t) \leq M * x_{i}(t) \quad \forall i \in I, \quad \forall t \in T \\
& \sum_{i=1}^{N_{1}} D R B_{j i}{ }^{\prime}(t) \leq M * y_{j}(t) \quad \forall j \in J, \quad \forall t \in T \\
& \sum_{j=1}^{N_{2}} D R B_{i j}(t) \geq x_{i}(t), \quad \forall i \in I, \forall t \in T \\
& \sum_{i=1}^{N_{1}} D R B_{j i}{ }^{\prime}(t) \geq y_{j}(t), \quad \forall j \in J, \quad \forall t \in T \\
& \sum_{j=1}^{N_{2}} L B_{i j}(t)=\min \left(O B_{i}(t), \sum_{j=1}^{N_{2}} x q_{i j}(t)\right), \quad \forall i \in I, \forall t \in T \\
& \sum_{i=1}^{N_{1}} L B_{j i}{ }^{\prime}(t)=\min \left(D B_{j}(t), \sum_{i=1}^{N_{1}} x q_{j i}{ }^{\prime}(t)\right), \quad \forall j \in J, \forall t \in T \\
& D R B_{i j}(t) \cdot D R B_{j i}{ }^{\prime}(t)=0, \text { if } x_{i}(t)=y_{j}(t)=1, \quad \forall i \in I, \forall j \in J, \forall t \in T \\
& \sum_{i=1}^{N_{1}} O B_{i}(t)+\sum_{j=1}^{N_{2}} D B_{j}(t)=\sum_{i=1}^{N_{1}} O B_{i}(1)+\sum_{j=1}^{N_{2}} D B_{j}(1), \quad \forall t \in T \\
& \sum_{i=1}^{N_{1}} \sum_{t=1}^{T_{\max }} x_{i}(t)+\sum_{j=1}^{N_{2}} \sum_{t=1}^{T_{\max }} y_{j}(t) \leq k \\
& \sum_{j=1}^{N_{2}} D R B_{i j}(t) \leq l \cdot x_{i}(t), \quad \forall t \in T, \quad \forall i \in I \\
& \sum_{i=1}^{N_{1}} D R B_{j i}{ }^{\prime}(t) \leq l \cdot y_{j}(t), \quad \forall t \in T, \forall j \in J \\
& x_{i}\left(T_{\max }\right)=0, \quad \forall i \in N_{1} \\
& y_{j}\left(T_{\max }\right)=0, \quad \forall j \in J \\
& O B_{i}(t) \geq 0 \text {, } \\
& D B_{j}(t) \geq 0 \text {, } \\
& L B_{i j}(t) \geq 0 \text {, } \\
& L B_{j i}{ }^{\prime}(t) \geq 0, \\
& D R B_{i j}(t) \geq 0 \text {, } \\
& D R B_{j i}{ }^{\prime}(t) \geq 0, \\
& U B_{i j}(t) \geq 0, \\
& U B_{j i}{ }^{\prime}(t) \geq 0 \text {, } \\
& x_{i} \in\{0,1\} \text {, } \\
& y_{j} \in\{0,1\} \text {, }
\end{aligned}
$$

Constraints (2) and (3) represent the number of bikes in each station obtained by taking into account the previous bike flows. Constraints (4) and (5) demonstrate that the number of unsatisfied demands in period $t+1$ equals the number of 
TABLE 2: Information of bidirectional trip demand for the example.

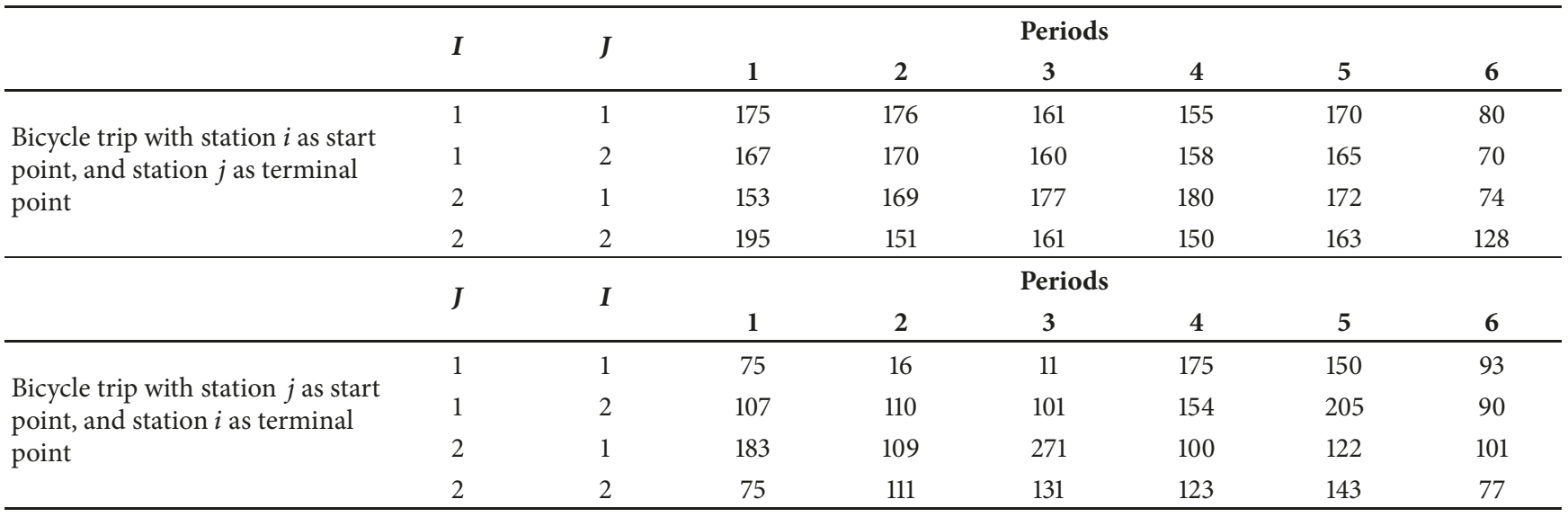

unmet demands from the previous period and the number of unmet demands from the current time period. Constraints (6) and (7) represent the fact that the unmet demand at the initial period of the planning horizon is determined by the deviation of the travel demand and the practical number of rented bikes. Constraints (8) and (9) represent the number of bikes that can be rented at each site, at the beginning of each time period. Constraints (10) and (11) represent the number of empty bikes that can be dispatched at each site, at the beginning of each time period. Constraints (12) and (13) ensure that the empty bikes are only moved at the dispatched stations and time periods that are determined. If the station does make a no-dispatching decision at a certain period, the number of unutilized bikes to be moved out must equal zero to satisfy these constraints. If the station makes a dispatching decision at a certain period, the number of unutilized bikes to be moved out is a nonnegative number and always satisfies these constraints because $\mathrm{M}$ is a sufficiently large positive constant. Constraints (14) and (15) represent the fact that once a dispatching determination is made at a site in a time period, then there must be bikes being dispatched. This means that if the station makes a transporting decision at a certain period, at least one empty bike is dispatched from that site. Constraints (16) and (17) avoid the situation that bikes cannot be rented with empty bike staying at the same site. Constraint (18) avoids the situation that convective transport movement of empty bikes is presented between two sites at the same time period. Constraint (19) represents the fact that all bikes in the system need to be allocated at sites. Constraint (20) represents the limit of dispatching frequencies in the planning horizon. Constraints (21) and (22) represent the capacity of each truck at each dispatching is carried. Constraints (23) and (24) represent the fact that no dispatching is carried out in the last period of planning horizon. Finally, decisions to set up dispatching are modeled by binary variables whereas all the other variables are nonnegative integers as in (25).

3.4. Solution Method. The aim of this model is to decide the fleet size of bikes in the system and to ensure proper dispatching time period and number, so as to realize a maximum bicycle travel trips. Since there are multiperiod demands and
TABLE 3: The input parameters for the example.

\begin{tabular}{lccc}
\hline $\begin{array}{l}\text { Input } \\
\text { parameters }\end{array}$ & value & $\begin{array}{c}\text { Input } \\
\text { parameters }\end{array}$ & value \\
\hline$h$ & 0.01 & $\beta$ & $¥ 0.2$ \\
$s_{i} / s_{j}$ & 400 & $\theta$ & $¥ 0.1$ \\
$b$ & $¥ 0.07$ & $p$ & $¥ 3$ \\
$k$ & 4 & $r$ & $¥ 1.5$ \\
$l$ & 50 & $M$ & 10000 \\
$f$ & $¥ 100$ & & \\
\hline
\end{tabular}

many constraint conditions, if the number of regional stations is large, the solution will become quite complex. This problem is a large-scale nonlinear integer programming model.

In this paper, we obtain the numerical solution to the optimization problem with LINGO, which is an object-oriented programming language. LINGO can be used to efficiently solve the linear, nonlinear, and integer programming model. Its solver features the advantages of fast solving speed, direct, and clear performance in optimizing objectives, constraining models, and calculating. The problem of this paper can be solved by the branch and bound approach of the LINGO solver.

\section{Numerical Example}

4.1. Problem Description. According to the operation and data of the FFBS in China, this paper designs and operates the model. In this example, two small regions are included with both regions owning 2 stations, i.e., $N_{1}=2, N_{2}=2$. Note that there is no limitation on the values of $N_{1}$ and $N_{2}$ in this model and $N_{1} \neq N_{2}$ can also be applied to them. In this example, the time horizon is 6 hours. We split the time horizon with the time interval being one hour, hence, $T_{\max }=$ 6. The information of bidirectional trip demand is shown in Table 2 and the input parameters are listed in Table 3.

4.2. Results. This part uses LINGO 11 to solve the integer nonlinear programming model with branch and bound approach. After running the LINGO program, the following data is 
TABLE 4: Results of the illustrative example.

\begin{tabular}{|c|c|c|c|c|c|c|c|c|}
\hline Periods & $L B_{i j}$ & $L B^{\prime}{ }_{j i}$ & $D R B_{j i}$ & $D R B_{j i}^{\prime}$ & $U B_{i j}$ & $U B^{\prime}{ }_{j i}$ & $O B_{i}$ & $D B_{j}$ \\
\hline \multirow[t]{4}{*}{$t=1$} & $L B_{11}=175$ & $L B_{11}^{\prime}=75$ & $D R B_{11}=0$ & $D R B_{11}^{\prime}=0$ & $U B_{11}=0(0)$ & $U B_{11}^{\prime}=0(0)$ & $O B_{1}=384$ & $D B_{1}=182$ \\
\hline & $L B_{12}=167$ & $L B_{12}^{\prime}=107$ & $D R B_{12}=0$ & $D R B^{\prime}{ }_{12}=0$ & $U B_{12}=0(0)$ & $U B^{\prime}{ }_{12}=0(0)$ & & \\
\hline & $L B_{21}=153$ & $L B^{\prime}{ }_{21}=183$ & $D R B_{21}=0$ & $D R B^{\prime}{ }_{21}=0$ & $U B_{21}=0(0)$ & $U B^{\prime}{ }_{21}=0(0)$ & $O B_{2}=348$ & $D B_{2}=258$ \\
\hline & $L B_{22}=195$ & $L B_{22}^{\prime}=75$ & $D R B_{22}=0$ & $D R B_{22}^{\prime}=0$ & $U B_{22}=0(0)$ & $U B_{22}^{\prime}=0(0)$ & & \\
\hline total & 690 & 440 & 0 & 0 & $0(0)$ & $0(0)$ & 732 & 440 \\
\hline \multirow[t]{4}{*}{$t=2$} & $L B_{11}=130$ & $L B_{11}^{\prime}=16$ & $D R B_{11}=0$ & $D R B_{11}^{\prime}=19$ & $U B_{11}=46(46)$ & $U B_{11}^{\prime}=0(0)$ & $O B_{1}=300$ & $D B_{1}=328$ \\
\hline & $L B_{12}=170$ & $L B_{12}^{\prime}=110$ & $D R B_{12}=0$ & $D R B^{\prime}{ }_{12}=31$ & $U B_{12}=0(0)$ & $U B_{12}^{\prime}=0(0)$ & & \\
\hline & $L B_{21}=42$ & $L B_{21}^{\prime}=109$ & $D R B_{21}=0$ & $D R B^{\prime}{ }_{21}=43$ & $U B_{21}=127(127)$ & $U B_{21}^{\prime}=0(0)$ & $O B_{2}=182$ & $D B_{2}=362$ \\
\hline & $L B_{22}=140$ & $L B_{22}^{\prime}=111$ & $D R B_{22}=0$ & $D R B_{22}^{\prime}=7$ & $U B_{22}=11$ & $U B_{22}^{\prime}=0(0)$ & & \\
\hline total & 482 & 346 & 0 & 100 & $184(184)$ & $0(0)$ & 482 & 690 \\
\hline \multirow[t]{4}{*}{$t=3$} & $L B_{11}=136$ & $L B_{11}^{\prime}=11$ & $D R B_{11}=0$ & $D R B_{11}^{\prime}=12$ & $U B_{11}=25(71)$ & $U B^{\prime}{ }_{11}=0(90)$ & $O B_{1}=187$ & $D B_{1}=324$ \\
\hline & $L B_{12}=51$ & $L B_{12}^{\prime}=101$ & $D R B_{12}=0$ & $D R B^{\prime}{ }_{12}=38$ & $U B_{12}=109(109)$ & $U B_{12}^{\prime}=0(0)$ & & \\
\hline & $L B_{21}=87$ & $L B^{\prime}{ }_{21}=271$ & $D R B_{21}=0$ & $D R B^{\prime}{ }_{21}=0$ & $U B_{21}=90(217)$ & $U B_{21}^{\prime}=0(0)$ & $O B_{2}=259$ & $D B_{2}=402$ \\
\hline & $L B_{22}=172$ & $L B_{22}^{\prime}=131$ & $D R B_{22}=0$ & $D R B^{\prime}{ }_{22}=0$ & $U B_{22}=0(0)$ & $U B^{\prime}{ }_{22}=0(0)$ & & \\
\hline total & 446 & 514 & 0 & 50 & $224(397)$ & $0(0)$ & 446 & 726 \\
\hline \multirow[t]{4}{*}{$t=4$} & $L B_{11}=174$ & $L B_{11}^{\prime}=175$ & $D R B_{11}=0$ & $D R B_{11}^{\prime}=0$ & $U B_{11}=0(52)$ & $U B_{11}^{\prime}=0(0)$ & $O B_{1}=294$ & $D B_{1}=385$ \\
\hline & $L B_{12}=120$ & $L B_{12}^{\prime}=154$ & $D R B_{12}=0$ & $D R B_{12}^{\prime}=0$ & $U B_{12}=38(147)$ & $U B^{\prime}{ }_{12}=0(0)$ & & \\
\hline & $L B_{21}=125$ & $L B^{\prime}{ }_{21}=100$ & $D R B_{21}=0$ & $D R B^{\prime}{ }_{21}=0$ & $U B_{21}=55(272)$ & $U B^{\prime}{ }_{21}=0(0)$ & $O B_{2}=270$ & $D B_{2}=223$ \\
\hline & $L B_{22}=145$ & $L B^{\prime}{ }_{22}=123$ & $D R B_{22}=0$ & $D R B^{\prime}{ }_{22}=0$ & $U B_{22}=5(5)$ & $U B_{22}^{\prime}=0(0)$ & & \\
\hline total & 564 & 552 & 0 & 0 & $98(476)$ & $0(0)$ & 564 & 608 \\
\hline \multirow[t]{4}{*}{$t=5$} & $L B_{11}=0$ & $L B^{\prime}{ }_{11}=150$ & $D R B_{11}=0$ & $D R B^{\prime}{ }_{11}=0$ & $U B_{11}=170(222)$ & $U B^{\prime}{ }_{11}=0(0)$ & $O B_{1}=275$ & $D B_{1}=355$ \\
\hline & $L B_{12}=275$ & $L B_{12}^{\prime}=205$ & $D R B_{12}=0$ & $D R B^{\prime}{ }_{12}=0$ & $U B_{12}=0(37)$ & $U B^{\prime}{ }_{12}=0(0)$ & & \\
\hline & $L B_{21}=183$ & $L B^{\prime}{ }_{21}=122$ & $D R B_{21}=0$ & $D R B^{\prime}{ }_{21}=0$ & $U B_{21}=0(261)$ & $U B^{\prime}{ }_{21}=0(0)$ & $O B_{2}=277$ & $D B_{2}=265$ \\
\hline & $L B_{22}=94$ & $L B_{22}^{\prime}=143$ & $D R B_{22}=0$ & $D R B_{22}^{\prime}=0$ & $U B_{22}=69(74)$ & $U B_{22}^{\prime}=0(0)$ & & \\
\hline total & 552 & 620 & 0 & 0 & $239(594)$ & $0(0)$ & 552 & 620 \\
\hline \multirow[t]{4}{*}{$t=6$} & $L B_{11}=43$ & $L B_{11}^{\prime}=93$ & $D R B_{11}=0$ & $D R B_{11}^{\prime}=0$ & $U B_{11}=37(259)$ & $U B_{11}^{\prime}=0(0)$ & $O B_{1}=272$ & $D B_{1}=183$ \\
\hline & $L B_{12}=107$ & $L B_{12}^{\prime}=90$ & $D R B_{12}=0$ & $D R B^{\prime}{ }_{12}=0$ & $U B_{12}=0(0)$ & $U B^{\prime}{ }_{12}=0(0)$ & & \\
\hline & $L B_{21}=0$ & $L B^{\prime}{ }_{21}=101$ & $D R B_{21}=0$ & $D R B^{\prime}{ }_{21}=0$ & $U B_{21}=74(335)$ & $U B^{\prime}{ }_{21}=0(0)$ & $O B_{2}=348$ & $D B_{2}=369$ \\
\hline & $L B_{22}=202$ & $L B_{22}^{\prime}=77$ & $D R B_{22}=0$ & $D R B_{22}^{\prime}=0$ & $U B_{22}=0(0)$ & $U B_{22}^{\prime}=0(0)$ & & \\
\hline total & 352 & 361 & 0 & 0 & $111(594)$ & $0(0)$ & 620 & 552 \\
\hline
\end{tabular}

acquired: $\eta=4588.73$, the total number of bikes is 1172 , the number of overload at stations is 2 , the number of unsatisfied travel demand is 594, the number of total scheduling is 150 , and the total number of traveling is 5919 (the average utilization rate is $5919 / 1172=5.05)$. The results are shown in Table 4.

In the columns of $U B_{i j}$ and $U B^{\prime}{ }_{j i}$, the number in the bracket is the accumulated number of unmet demands that is added up to the current time period; the number outside the bracket is the unmet demands at the current time period.

It is worth noting that, under the optimal target value, the allocation number of stations is not exclusive. There exists nuance at different time periods. But the total number of deployed bikes and the overload in the system is fixed as well as the optimal objective function value.

\subsection{Extensions and Discussions}

4.3.1. Time Expanded Model. In real life, the planning horizon may be longer and the time division may be smaller, e.g., half an hour or 15 minutes as an interval point. In this context, the service and movement time may be longer than a unit interval; thus the time period can be extended on the basis of the original model, for instance, to replace the flow of starting from $i$ to $j$ during time $t$ to time $t+1$ with the flow of starting from $i$ to $j$ during time $t$ to time $t+2$. Furthermore, the average utilization rate per bike in a longer planning horizon and the economic feasibility of system can be further discussed.

4.3.2. Discussion on the Average Utilization Rate per Bike and Quantity of Deployment. In the whole planning horizon, the average utilization rate per bike and the economic feasibility are inextricably tied. Therefore, the relationship of these two factors in the model is worth observing.

Denote the utilization rate per bike at time period $t$ by $\alpha(t)$. We have

$$
\alpha(t)=\frac{\sum_{i=1}^{N_{1}} \sum_{j=1}^{N_{2}}\left(L B_{i j}(t)+L B_{j i}{ }^{\prime}(t)\right)}{\sum_{i=1}^{N_{1}} O B_{i}(1)+\sum_{j=1}^{N_{2}} D B_{j}(1)} .
$$




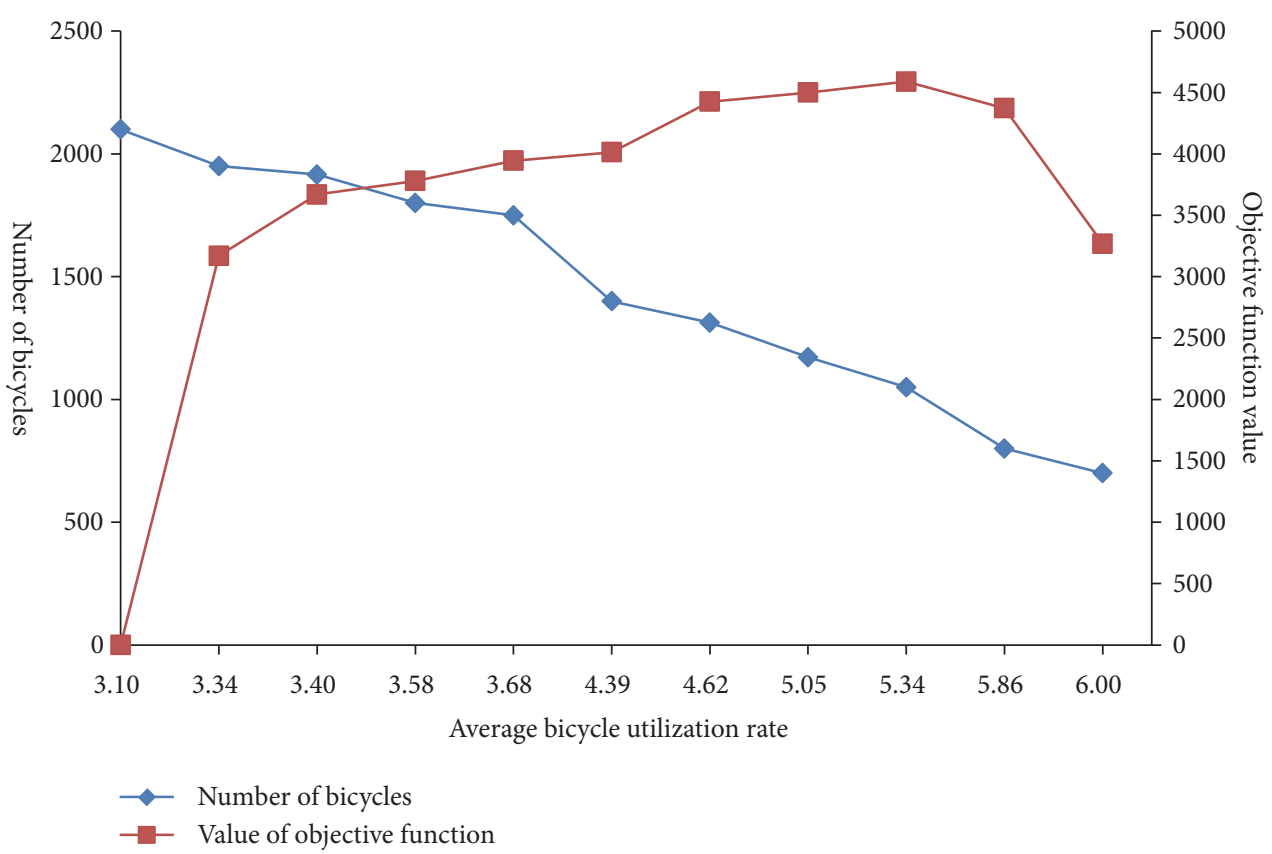

FIGURE 3: Effect of the average utilization rate per bike on FFBS system.

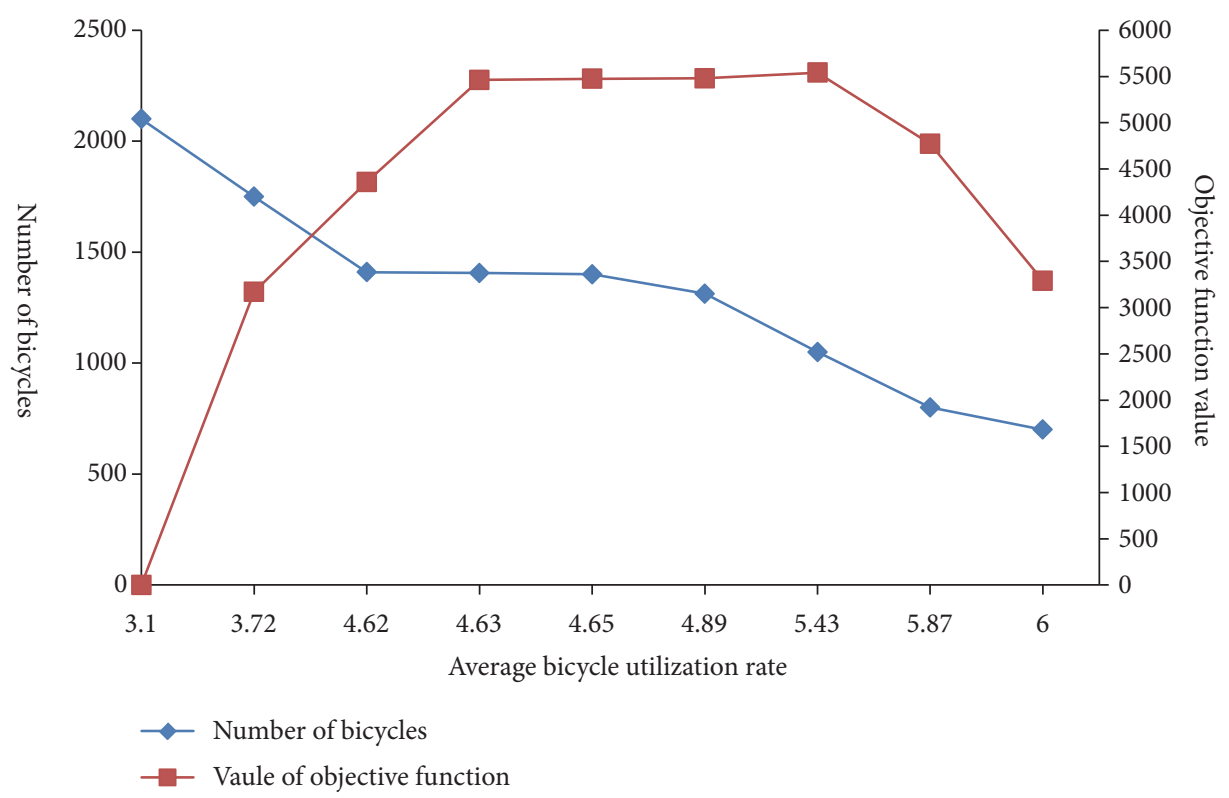

FIGURE 4: Effect of the average utilization rate per bike (without the constraints of truck capacity and startup cost) on FFBS system.

Let $u_{\beta}$ be the average utilization rate per bike in the whole planning horizon. Thus,

$$
u_{\beta}=\sum_{t=1}^{T_{\max }} \alpha(t) .
$$

On the basis of this definition, an analog observation on $u_{\beta}$, as well as the quantity of deployment and the income of the system, is carried out. The results are shown in Figure 3.

If there is no limit on the load capacity of truck dispatching, the constraints (21) and (22) can be canceled; meanwhile no startup cost is set, which means that the startup cost is zero so that the implicit dispatching frequencies will not affect the system. The final objective function value is 5542.27 ; the number of deployed bike is 1313 (total turnover rate is $6420 / 1313=4.89$ ); the total number of overload is 75 ; the total number of scheduling is 510 ; and the number of unsatisfied demand is 93. Meanwhile, an analogy observation of the modified model as well as the number of allocated bikes and revenue of the system is carried out. The results are shown in Figure 4. 
Figures 3 and 4 illustrate that the number of deployed bikes is inversely proportion to the utilization rate; i.e., the less bikes are deployed, the higher average utilization rate can be reached. To ensure the economic feasibility of the system operation, the utilization rate per bike should be higher than a particular threshold; and to ensure a relevantly impressive income, more bikes should be deployed to accumulate more travel; however, this will reduce the average utilization rate per bike. Hence, a subtle balance between system revenue and utilization rate per bike is necessary to be explored.

\section{Conclusions}

The proper fleet dimensioning and dispatching problem is an important problem in the operation of FFBS systems because it prominently affects the performance and efficiency of the systems. This paper proposed an optimization model for the deployment and travel of FFBS among small regions. The problem is to decide a certain number of bikes for deployment and to determine the proper period and the number of bikes to be redistributed that will satisfy the need of operators for obtaining maximum profit during overall period. A numerical example solved by LINGO solver was given to illustrate the proposed model.

The contributions of this paper are as follows. (i) Most of the literature studies focused on SBBS systems. The proposed model can determine the number of bikes in an FFBS system and the number of initial bikes at each station and the movement time point of unutilized bikes during the overall period. (ii) Instead of only discussing static repositioning, the proposed model implements a multiperiodic dynamic analysis to meet the demand of users for bikes at each station of an FFBS system in small regions. (iii) The model in this study introduces penalty costs in an FFBS system for overload of flexible stations.

Future studies may take into account uncertain demand and the service order of the demands in the optimizing deployment of an FFBS system. Moreover, vehicle routing problems need to be taken into account when considering movements of unutilized bikes among stations in operation planning of FFBS.

\section{Data Availability}

The data used to support the findings of this study are included within the article.

\section{Conflicts of Interest}

The authors declare no conflicts of interest.

\section{Acknowledgments}

This work was supported by the National Natural Science Foundation of China [Grant nos. 71401164, 71601014], the Soft Science Foundation of Hebei Province [Grant no. 18454204D], and the Program for Sciences and Technology of Hebei province [Grant nos. 164576484, 17454209], and the Fundamental Research Funds for the Central Universities [Grant no. 2018RC023].

\section{References}

[1] CAICT, "The Development of China's free-floating shared bicycle Industry," Tech. Rep., Beijing, China, 2018, http://www.caict .ac.cn/kxyj/qwfb/ztbg/201804/P020180320523449466152.pdf.

[2] D. K. George and C. H. Xia, "Fleet-sizing and service availability for a vehicle rental system via closed queueing networks," European Journal of Operational Research, pp. 198-207, 2011.

[3] J. Shu, M. C. Chou, Q. Liu, C. P. Teo, and I. L. Wang, "Models for effective deployment and redistribution of bicycles within public bicycle-sharing systems," Operations Research, vol. 61, no. 6, pp. 1346-1359, 2013.

[4] C. Fricker and N. Gast, "Incentives and redistribution in homogeneous bike-sharing systems with stations of finite capacity," Euro Journal on Transportation and Logistics, vol. 5, no. 3, pp. 261-291, 2016.

[5] J. R. Lin and T. H. Yang, "Strategic design of public bicycle sharing systems with service level constraints," Transportation Research Part E: Logistics and Transportation Review, vol. 47, no. 2, pp. 284-294, 2011.

[6] J. R. Lin, T. H. Yang, and Y. C. Chang, "A hub location inventory model for bicycle sharing system design: Formulation and solution," Industrial Engineering, vol. 65, no. 1, pp. 77-86, 2013.

[7] T. Raviv and O. Kolka, "Optimal inventory management of a bike-sharing station," IIE Transactions, vol. 45, no. 10, pp. 10771093, 2013.

[8] T. Raviv, M. Tzur, and I. A. Forma, "Static repositioning in a bike-sharing system: models and solution approaches," EURO Journal on Transportation and Logistics, vol. 2, no. 3, pp. 187229, 2013.

[9] R. Nair and E. Miller-Hooks, "Fleet management for vehicle sharing operations," Transportation Science, vol. 45, no. 4, pp. 524-540, 2011.

[10] J. Schuijbroek, R. C. Hampshire, and W.-J. van Hoeve, "Inventory rebalancing and vehicle routing in bike sharing systems," European Journal of Operational Research, vol. 257, no. 3, pp. 992-1004, 2017.

[11] P. Vogel, B. A. N. Saavedra, and D. C. Mattfeld, "A hybrid metaheuristic to solve the resource allocation problem in bike sharing systems," in Proceedings of the International Workshop on Hybrid Metaheuristics, pp. 16-29, Springer, June 2014.

[12] P. Vogel, "Service network design of bike sharing systems," in Service Network Design of Bike Sharing Systems, pp. 113-135, Springer, 2016.

[13] B. A. Neumann-Saavedra, P. Vogel, and D. C. Mattfeld, "Anticipatory service network design of bike sharing systems," Transportation Research Procedia, vol. 10, pp. 355-363, 2015.

[14] B. A. Neumann-Saavedra, T. G. Crainic et al., "Service network design of bike sharing systems with resource constraints," in Proceedings of the International Conference on Computational Logistics, pp. 352-366, Springer, 2016.

[15] H. Sayarshad, S. Tavassoli, and F. Zhao, "A multi-periodic optimization formulation for bike planning and bike utilization," Applied Mathematical Modelling, vol. 36, no. 10, pp. 4944-4951, 2012.

[16] S. Reiss and K. Bogenberger, “GPS-Data Analysis of Munich's Free-Floating Bike Sharing System and Application of an Operator-based Relocation Strategy," in Proceedings of the 18th 
IEEE International Conference on Intelligent Transportation Systems, pp. 584-589, September 2015.

[17] S. Weikl and K. Bogenberger, "Relocation strategies and algorithms for free-floating car sharing systems," Intelligent Transportation Systems Magazine, vol. 5, no. 4, pp. 100-111, 2013.

[18] A. Pal and Y. Zhang, "Free-floating bike sharing: solving reallife large-scale static rebalancing problems," Transportation Research Part C: Emerging Technologies, vol. 80, pp. 92-116, 2017.

[19] Y. Liu, W. Y. Szeto, and S. C. Ho, "A static free-floating bike repositioning problem with multiple heterogeneous vehicles, multiple depots, and multiple visits," Transportation Research Part C: Emerging Technologies, vol. 92, pp. 208-242, 2018.

[20] L. Caggiani, M. Ottomanelli, R. Camporeale, and M. Binetti, "Spatio-temporal clustering and forecasting method for freefloating bike sharing systems," in Proceedings of the International Conference on Systems Science, pp. 244-254, Springer, September 2016.

[21] L. Caggiani, R. Camporeale, M. Ottomanelli, and W. Y. Szeto, "A modeling framework for the dynamic management of freefloating bike-sharing systems," Transportation Research Part C: Emerging Technologies, vol. 87, pp. 159-182, 2018.

[22] L. Caggiani, R. Camporeale, and M. Ottomanelli, "Planning and Design of Equitable Free-Floating Bike-Sharing Systems Implementing a Road Pricing Strategy," Journal of Advanced Transportation, vol. 2017, Article ID 3182387, 13 pages, 2017.

[23] Z. Liu, Y. Shen, and Y. Zhu, "Inferring dockless shared bike distribution in new cities," in Proceedings of the the Eleventh ACM International Conference on Web Search and Data Mining, pp. 378-386, February 2018.

[24] Y. Ai, Z. Li, M. Gan et al., "A deep learning approach on shortterm spatiotemporal distribution forecasting of dockless bikesharing system," Neural Computing and Applications, pp. 1-13, 2018. 


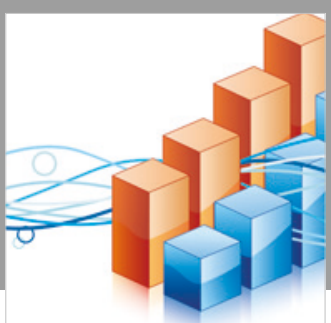

Advances in

Operations Research

\section{-n-m}
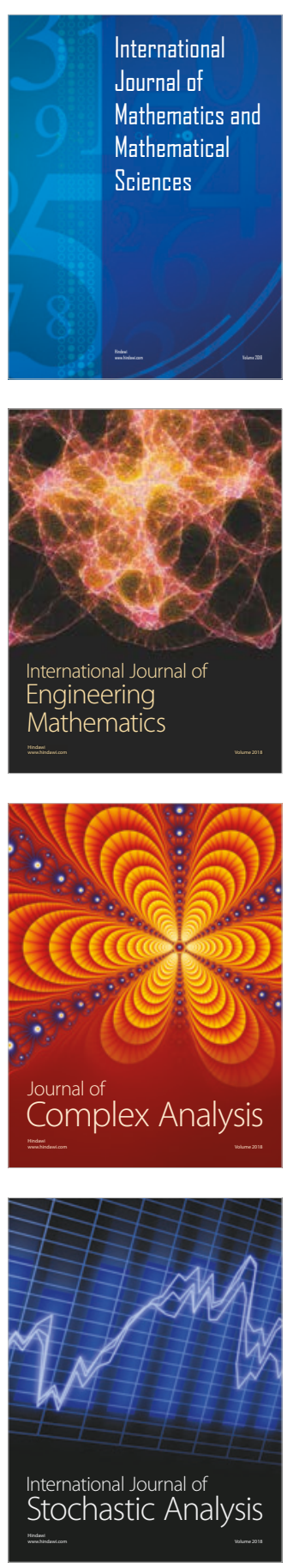
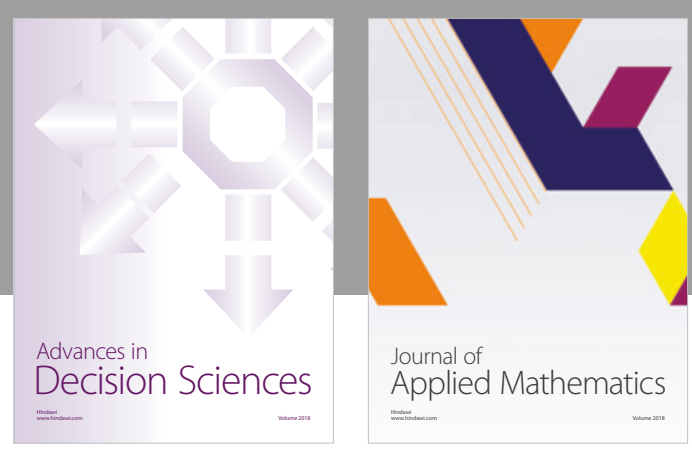

Journal of

Applied Mathematics
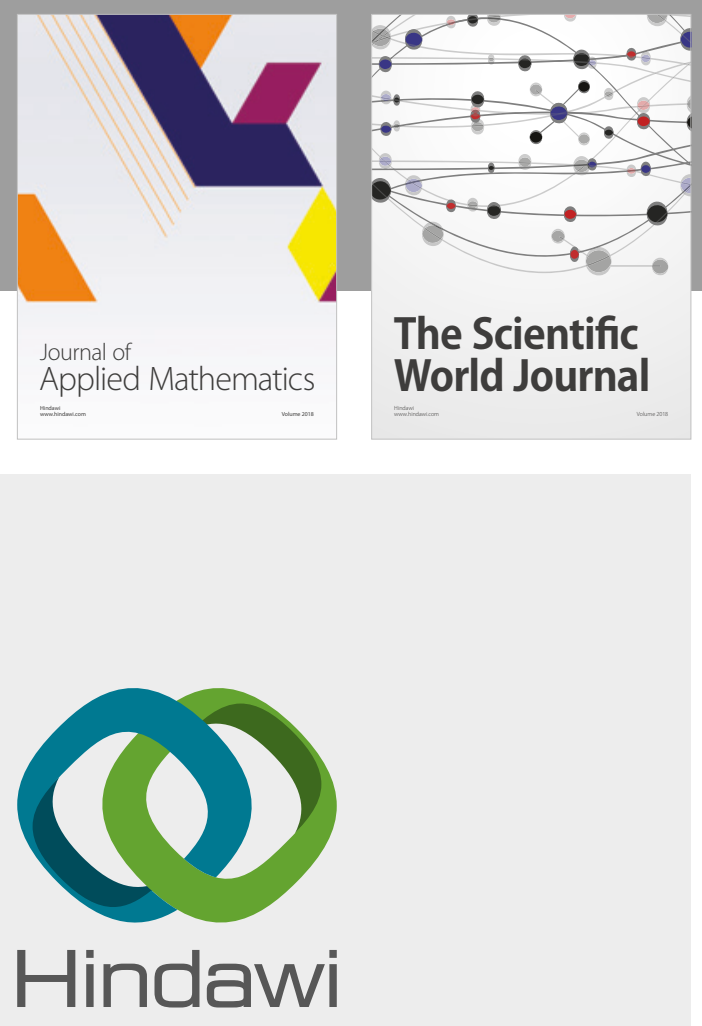

Submit your manuscripts at

www.hindawi.com

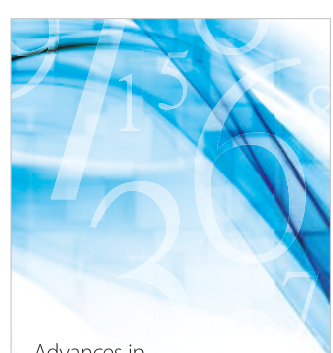

Advances in
Numerical Analysis
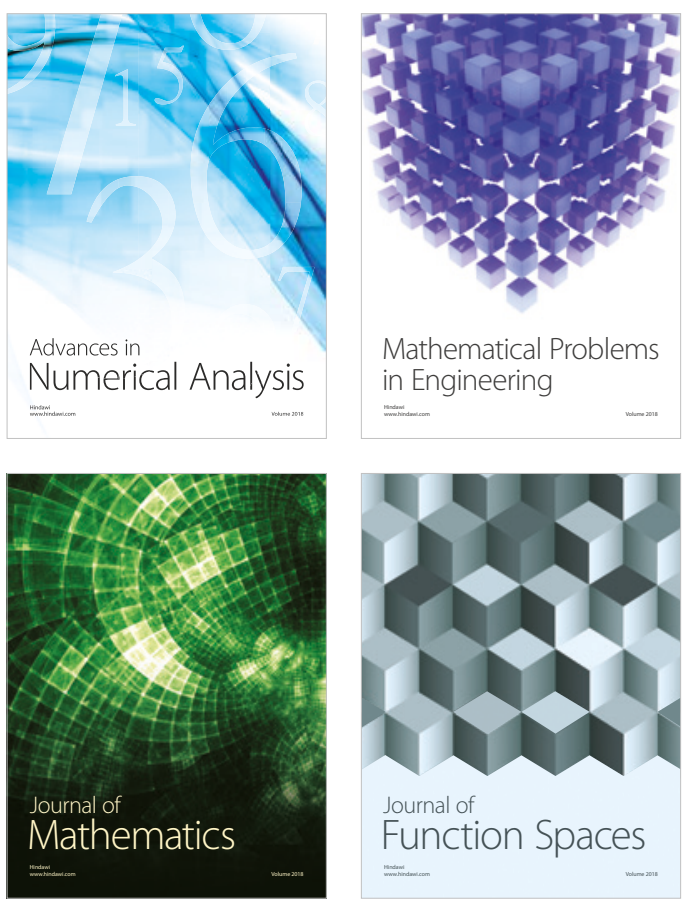

Mathematical Problems in Engineering

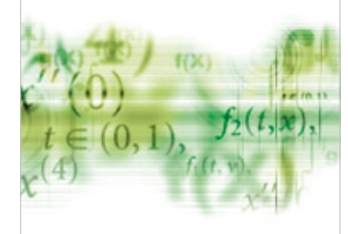

International Journal of

Differential Equations

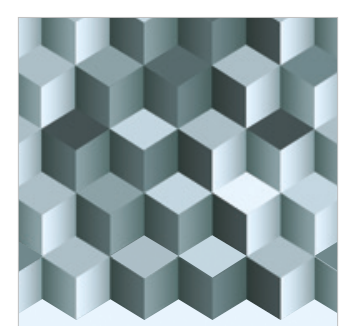

Journal of

Function Spaces

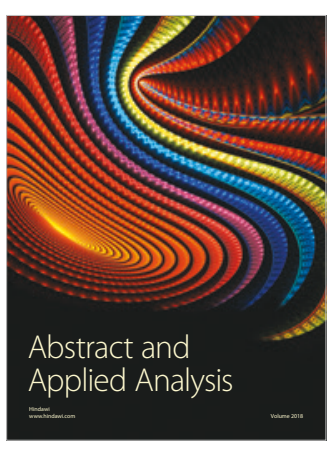

The Scientific

World Journal

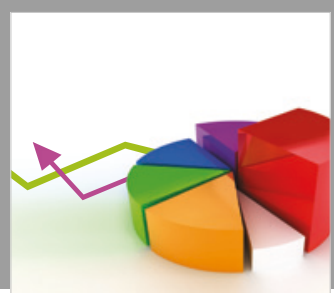

Journal of

Probability and Statistics
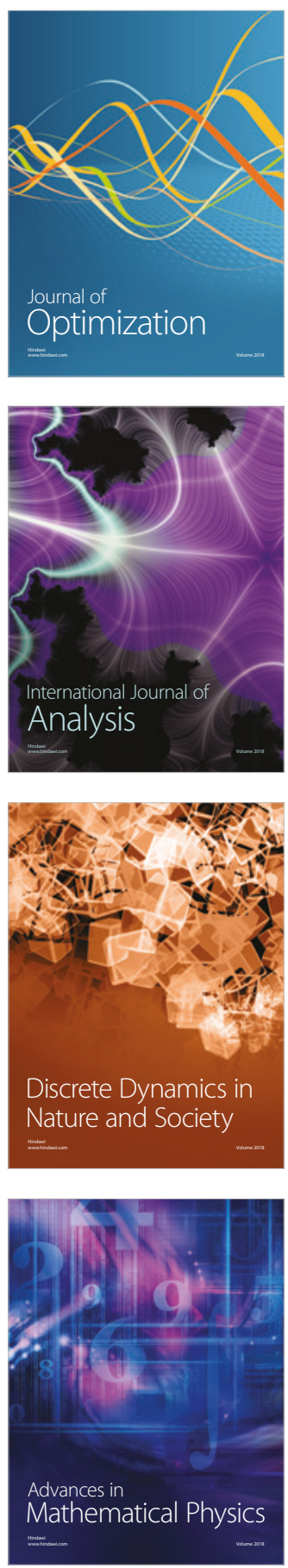